\title{
Knockdown of ANGPTL4 May Reduce the Expression of LPL during Differentiation of 3T3-L1 Preadipocyte
}

\author{
Zhao $\mathrm{X}^{1,2}$, Zhang $\mathrm{F}^{1}$, Liu $\mathrm{Y}^{1}$, Liu $\mathrm{H}^{1}$, Ao $\mathrm{H}^{3}$, Xing $\mathrm{K}^{4 *}$ \\ and Wang $\mathrm{C}^{1 *}$ \\ ${ }^{1}$ National Engineering Laboratory for Animal Breeding \\ and MOA Key Laboratory of Animal Genetics and \\ Breeding, Department of Animal Genetics and Breeding, \\ China Agricultural University, Beijing, China \\ ${ }^{2}$ Beijing Shunxin Agriculture Co., Ltd., Beijing, China \\ ${ }^{3}$ The State Key Laboratory of Animal Nutrition, Institute \\ of Animal Sciences, Chinese Academy of Agricultural \\ Sciences, Beijing, China \\ ${ }^{4}$ Animal Science and Technology College, Beijing \\ University of Agriculture, Beijing, China \\ *Corresponding author: Kai Xing, National \\ Engineering Laboratory for Animal Breeding and MOA \\ Key Laboratory of Animal Genetics and Breeding, \\ Department of Animal Genetics and Breeding, China \\ Agricultural University, Beijing, China \\ Chuduan Wang, National Engineering Laboratory for \\ Animal Breeding and MOA Key Laboratory of Animal \\ Genetics and Breeding, Department of Animal Genetics \\ and Breeding, China Agricultural University, Beijing, \\ China
}

Received: March 05, 2021; Accepted: March 25, 2021; Published: April 01, 2021

\section{Introduction}

The main form of fat is triglyceride, and the proportion of up to $95 \%$, in addition to lipid, fat also contain water and protein [1]. There are two main types of adipose tissue in mammals: white fat and brown fat. The function of white adipose tissue is to convert fat into energy to meet the needs of the body, and it is also the adipose tissue that is mainly amplified when getting fat. The function of brown adipose tissue is to generate heat, mainly through mitochondria to break down fatty acids to produce free fatty acids for energy [2]. Adipose deposition is mainly determined by the expansion of adipose tissue, which in turn is determined by the increase in the volume and number of adipocytes. Either way, the formation of adipocyte is a crucial step. Adipose tissue is a complex, highly metabolically active tissue involved in the regulation of systemic energy balance [3]. The expansion of adipose tissue is mainly affected by two processes: the number of adipocytes and the change of adipocyte size [4]. During adipogenesis, pluripotent Mesenchymal Stem Cells (MSCs) first develop into adipoblasts, which then differentiate into preadipocytes, and finally, the preadipocytes differentiate into adipocytes under certain conditions [5]. The number of mature adipocytes in adipose tissue is considered to be a marker of preadipocyte proliferation and subsequent differentiation into mature adipocytes [6]. Adipocyte differentiation is regulated by multiple factors. For example, TNF- $\alpha$ has been found to inhibit adipocyte differentiation [7], and C/EBPa can regulate adipocyte terminal differentiation [8]. PPAR $\gamma$ can induce adipocyte generation [9], and SIRT 1 can negatively regulate PPAR $\gamma$ to inhibit adipocyte differentiation [10]. In addition, scientists have also found that FoxO regulates adipocyte differentiation and can interact with SIR T1 to regulate adipocyte differentiation [11].

The 3T3-L1 preadipocyte line was cloned from Swiss 3T3 mouse embryos by Green and Kehinde in 1976 and has the potential to differentiate into mature adipocytes in vitro. Even after implantation into animals, the 3T3-L1 preadipocyte line can differentiate into normal adipocytes [12]. Due to this significant advantage, the 3T3-L1 preadipocyte line has become the most widely studied cell line for adipocyte proliferation, differentiation and regulation of related genes [13]. Compared with 3T3-L1 preadipocytes, primary preadipocytes need to be obtained from young animals, which requires a large amount of tissue, is difficult to separate, and is easy to be mixed with blood cells and other impurities. In addition, in order to ensure the differentiation specificity of the preadipocytes, the preadipocytes can only be transmitted within 5 generations. Therefore, the 3T3-L1 preadipocyte line has more extensive research and application value in the research that requires a large number of cells, such as functional verification and mechanism analysis. In recent years, more and more studies have been conducted to analyze the functional mechanisms of genes in 3T3-L1 cell lines. For example, researchers stimulated 3T3-L1 cell lines with quercetin and found that the anti-obesity effect of quercetin is mediated by AMPK and MAPK signaling pathways [14]. Both siRNA interference and overexpression vector are common test methods. For example, scientists knocked out the HMGA2 gene in 3T3-L1 cell line and 
found that differentiation of 3T3-L1 cell line was inhibited, indicating that let-7 plays an important role in adipocyte differentiation, partly by targeting HMGA2 to regulate the adipogenesis process [15]. MiR10b-5p regulates 3T3-L1 cell differentiation by targeting APOL6 [16]. Scientists used pcDNA3.1-GAS5 expression vector and GAS5siRNAs to overexpress and knockout GAS5, respectively, and found that GAS5 may indirectly increase the expression of phosphatase and tension protein homologous protein (PTEN) by inhibiting miRNA regulation mechanism of miR-21a-5p. In conclusion, GAS5 has an inhibitory effect on adipogenesis in 3T3-L1 cells, further emphasizing the importance of lncRNAs in adipogenesis [17]. Scientists found that Kisspeptin-10 inhibits the proliferation of 3T3-L1 cells and regulates lipid metabolism [18].

Angiopoietin-like protein 4 (ANGPTL4) is a secretory glycoprotein and a multipotent cytokine that is involved in angiogenesis and metastasis [19]. Recent studies have shown that ANGPTL4 has functions of regulating oncogenesis [20], vascular permeability [21], glucose homeostasis [22], lipid metabolism [23], energy homeostasis [24], wound healing and inflammation $[25,26]$. ANGPTL4 is also considered as an adipokine, which is involved in lipid metabolism and is highly expressed in adipose tissue and liver [27]. ANGPTL4 was highly expressed in pig adipose tissue, followed by liver, intestine and kidney [28]. ANGPTL4 is also related to muscles. Studies have shown that during fasting, the expression of ANGPTL4 in muscles increases and is involved in regulation of energy consumption through selective inhibition of LPL in resting muscles [29]. ANGPTL4 is involved in multiple fat deposition pathways through positive regulation of lipolysis and inhibition of Lipoprotein Lipase (LPL), which may have an important effect on livestock and poultry production traits [30]. A new study examined whether and how obesity alters a mother's blood Triglyceride (TG) levels during pregnancy to ensure a supply of lipids to the fetus. Maternal obesity in the trial was established by Pregnancy HF Feeding (PPHF), avoiding the influence of diet during pregnancy. In PPHF, ectopic overexpression of ANGPTL4 can restore maternal blood triglyceride concentration. These results suggest that ANGPTL4 plays a crucial role in increasing maternal triglyceride concentration during pregnancy. Obesity impairs maternal triglyceride concentration by decreasing the expression of ANGPTL4 in mice [31]. In our previous study, we found that ANGPTL4 is involved in fat deposition by participating in PPAR signaling pathway [32]. However, the exact mechanism is unclear. In this study, we observed the changes in the expression levels of key genes before and after ANGPTL4 interference to explore the specific mechanism of ANGPTL4's influence on lipid metabolism

\section{Materials and Methods}

\section{Culture of cells and induce of cells}

The 3T3-L1 preadipocyte were donated by the Institute of Animal Sciences, Chinese Academy of Agricultural Sciences and maintained in DMEM/F12 (Gibco, Carlsbad, CA, USA) supplied with 10\% FBS (Gibco, Carlsbad, CA, USA) at $5 \% \mathrm{CO}_{2}$ and $37^{\circ} \mathrm{C}$. When the cell confluence reaches $70 \%-80 \%$, cells were passaged by trypsin with 0.05\% EDTA (Gibco, Carlsbad, CA, USA). Then cells were planted in 6 wells plates (Corning Costar, New York, NY, USA) for inducing the 3T3-L1 differentiation. After reaching 90\% confluence, the standard culture medium was removed and replaced with differentiation medium which contained $10 \%$ FBS, $0.5 \mathrm{mM}$ 3-isobutyl-1methylxanthine, $1 \mu \mathrm{M}$ dexamethasone, and $10 \mu \mathrm{g} / \mathrm{mL}$ insulin (all Sigma, Beijing, China). After two days treatment, the differentiation medium was switched to maintenance medium (DMEM containing $10 \%$ FBS and $10 \mu \mathrm{g} / \mathrm{mL}$ insulin). Cell samples were collected on days 0, 2, 4 and 8, lysed using TRIzol reagent (Invitrogen, Carlsbad, CA, USA), and then stored in liquid nitrogen until RNA purification. There were three biological replicates per time point $(n=3)$.

\section{Design of SiRNA}

According to the sequence of mouse ANGPTL4 gene in GeneBank, three pairs of siRNA targeting and corresponding Negative Control was designed and synthesized by GenePharma (Suzhou, Jiangsu, China) (Table 1). The siRNA was centrifuged at $10000 \mathrm{rpm} / \mathrm{min}$ for $2 \mathrm{mins}$ and dissolved in $125 \mu \mathrm{L}$ of DEPC water to a final concentration $(20 \mu \mathrm{mol} / \mathrm{L})$.

\section{siRNA transfection and induce of cells}

When the cell confluence reaches 70-80\%, Lipofectamin 2000 (Invitrogen, Carlsbad, CA, USA) was used for transfection. According to the manufacturer's instructions, the medium was replaced with $2.5 \mathrm{ml}$ DMEM/F12 medium without serum and antibody 30 minutes before transfection. Then $5 \mu \mathrm{L}$ Lipofectamin 2000 for each well was taken and diluted with $250 \mu \mathrm{L}$ serum and double antibody free DMEM/F12 medium, mixed them gently, and incubated at room temperature for 5 minutes, name as mixture 1. $10 \mu \mathrm{L}$ siRNA was taken from each well, diluted with $250 \mu \mathrm{L}$ serum-free and doubleresistant DMEM/F12 medium, mixed gently, and incubated at room temperature for 5 minutes, name as mixture II. Mix the mixture 1 and 2 gently, standing $20 \mathrm{mins}$ under room temperature. Then add $500 \mu \mathrm{L}$ compound to each well, mix well. After incubating at $37^{\circ} \mathrm{C}$ for 6 hours, change the medium to complete medium. Then follow the steps above to induce cells.

\section{Oil red $O$ staining}

As previously described [32], well differentiated 3T3-L1 cells were washed with Phosphate-Buffered Saline (PBS) three times. Then the cells were fixed in $10 \%$ formaldehyde for $15 \mathrm{~min}$ and washed with PBS again. Next, cells were stained with $5 \%$ Oil Red O (Sigma, St. Louis, MO, USA) for 20min, washed twice with PBS. Finally, cells were photographed using an inverted microscope (Leica, Wetzlar, Germany).

\section{RNA extraction, chose the key genes and Quantitative qRT-PCR}

Total RNA was extracted by TRIzol regent (Invitrogen) and Table 1: Sequences of siRNA for mouse ANGPTL4.

\begin{tabular}{|l|c|c|}
\hline \multicolumn{1}{|c|}{ Sequence name } & & Sequence (5'-3') \\
\hline \multirow{2}{*}{ ANGPTL4-Mus-752 } & F & GGGACUGCCAGGAACUCUUTT \\
\cline { 2 - 3 } & $\mathrm{R}$ & AAGAGUUCCUGGCAGUCCCTT \\
\hline \multirow{2}{*}{ ANGPTL4-Mus-398 } & $\mathrm{F}$ & GCAUGGCUGCCUGUGGUAATT \\
\hline \multirow{2}{*}{ ANGPTL4-Mus-1376 } & $\mathrm{R}$ & UUACCACAGCCAGCCAUGCTT \\
\hline \multirow{2}{*}{ Negative control } & $\mathrm{F}$ & CCCUGCUGAUCCAGCCCAUTT \\
\hline & $\mathrm{R}$ & AUGGGCUGGAUCAGCAGGGTT \\
\hline & $\mathrm{F}$ & UUCUCCGAACGUGUCACGUTT \\
\hline
\end{tabular}


Table 2: Primers for quantitative real-time $P C R$.

\begin{tabular}{|c|c|c|}
\hline Gene & & Sequence (5'-3') \\
\hline \multirow{2}{*}{ ANGPTL4 } & $\mathrm{F}$ & GCATGGCTGCCTGTGGTAAC \\
\hline & $\mathrm{R}$ & ATCTTGCTGTTTTGAGCCTTGA \\
\hline \multirow{2}{*}{ PPARG } & $\mathrm{F}$ & GATTTCTCCAGCATTTCCA \\
\hline & $\mathrm{R}$ & GCTCTTCGTGAGGTTTGG \\
\hline \multirow{2}{*}{ ACAA2 } & $\mathrm{F}$ & TGAAATCAAGACCCTTGGGGG \\
\hline & $\mathrm{R}$ & GTACCCACAGCAAGGACACT \\
\hline \multirow{2}{*}{ SLC27A1 } & $\mathrm{F}$ & ACCCTGAAACACTGGAGGTC \\
\hline & $\mathrm{R}$ & CAGAGGCAAGCCTGGATGTA \\
\hline \multirow{2}{*}{ SCD } & $\mathrm{F}$ & TAGTCCCCAGCACGACTGTA \\
\hline & $\mathrm{R}$ & GCCATTGCCATCACCACAAG \\
\hline \multirow{2}{*}{ FASN } & $\mathrm{F}$ & GGCCAACTGGTCCTAAGCAT \\
\hline & $\mathrm{R}$ & СССTCTCTAGGGTGTGTCCA \\
\hline \multirow{2}{*}{ LPL } & $\mathrm{F}$ & AGCAGTTGAAGGCTGACTCC \\
\hline & $\mathrm{R}$ & GCTAGGCAGATGCCAGTTCA \\
\hline \multirow{2}{*}{ GADPH } & $\mathrm{F}$ & АСТСАСТСТTСТАССТTTGATGCT \\
\hline & $\mathrm{R}$ & TGTTGCTGTAGCCAAATTCA \\
\hline
\end{tabular}

reversely transcribed into cDNA according to the manufacturer's instructions. The reverse transcription quantitative PCR (RT-qPCR) was performed with the Light Cycler 480 Real-Time PCR system (Roche, Hercules, CA, USA). According to our previous research [32], ANGPTL4 was involved in the PPAR signaling pathway and has same expression pattern with ACAA2 and SLC27A1. Furthermore, we performed interaction analysis using STRING online software and found that ANGPTL4 was related to PPARG, LPL, FASN, SCD (Figure 1). Above all, we chose to observe the change of genes PPARG, SLC27A1, LPL, FASN, SCD expression before and after the interference of ANGPTL4. The primers used for the RT-qPCR detection of selected genes are listed in Table 2. All RT-qPCR experiments were carried out on three biological replicates with three technical replicates for every sample.

\section{Data analysis}

All quantitative results are presented as mean \pm standard deviation (SD). Statistical analyses were conducted using SPSS 20.0 software
(IBM, Almond, NY, USA). $\mathrm{P}<0.01$ was considered to be extremely significant, $\mathrm{p}<0.05$ was considered to be significant difference.

\section{Results}

\section{ANGPTL4 is Up-Regulated During 3T3-L1 Preadipocyte Differentiation}

To explore the role of ANGPTL4 in adipogenesis, we used 3T3L1 cells as the cell model. Firstly, we naturally induced 3T3-L1 cell differentiation. The mRNA levels of key genes were detected. The result showed that these genes included ANGPTL4 were increased gradually during adipogenesis (Figure 2). These results indicated that ANGPTL4 might play an important role in 3T3-L1 proliferation and differentiation.

\section{Efficiency of siRNA}

In order to further analyze the effect of ANGPTL4 on lipid metabolism, we conducted interference experiment on ANGPTL4 in 3T3-L1 preadipocyte. The interference results show that efficiency of si-ANGPTL4-752 is the highest, up to $90 \%$ (Figure 3). So, we used the si-ANGPTL4-752 for the next step.

\section{Effect of ANGPTL4 on the expression of key genes}

To explore how ANGPTL4 influence 3T3-L1 differentiation, we chose observe change of ACAA2, SLC27A1, SCD, FASN, LPL, PPARG expression before and after inhibiting ANGPTL4. The results showed that, interference of ANGPTL4 did not block the adipocyte differentiation process (Figure 4). Expression of all genes increased gradually before inhibited ANGPTL4. However, after silencing ANGPTL4, the expression levels of all genes except LPL were significantly increased first and then rapidly decreased. Furthermore, the expression pattern of LPL was similar with ANGPTL4 which decreased from day 2 to day 4 and then increased from day 4 to day 8 (Figure 5).

\section{Discussion}

The regulation of lipid metabolism is a complex and dynamic balance [33]. Lipid metabolism is also an important factor in maintaining health and determining fat deposition [34]. The $\mathrm{N}$-terminus of AngPTL4 mediates the inhibition of LPL activity, while the C-terminus stimulates adipocyte lipolysis, releasing stored TGS into the blood in the form of fatty acids and glycerol, further reducing
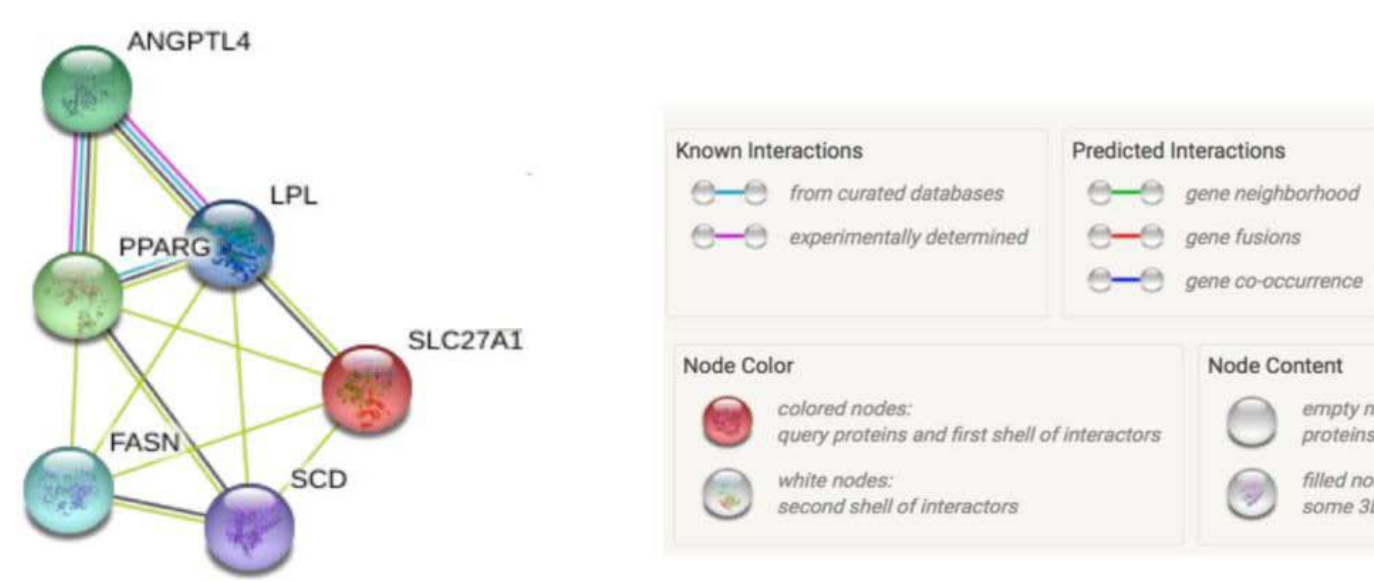

Others

- -1 textmining

-9 co-expression

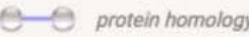

Node Content

Figure 1: Network of ANGPTL4 and its related genes involved in lipid metabolism. 


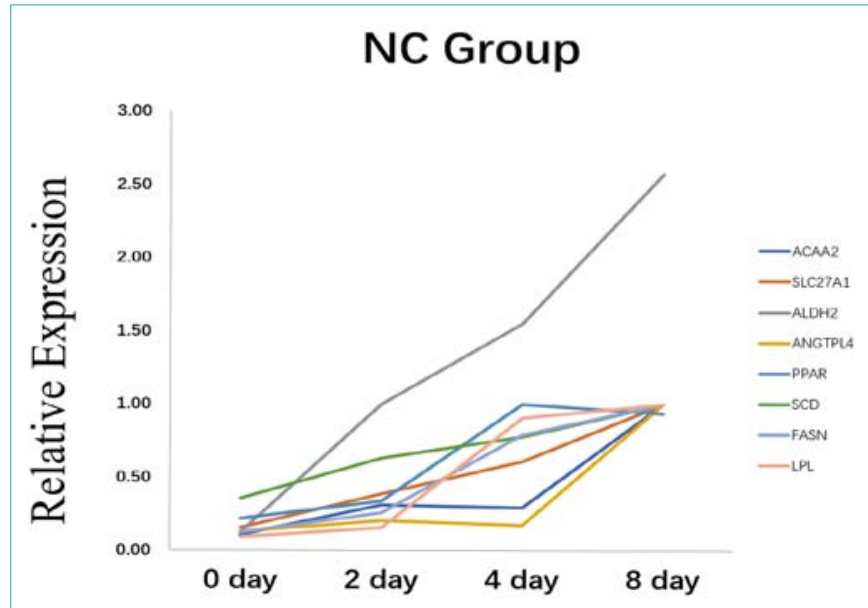

Figure 2: The mRNA relative expression level of ANGPTL4 and adipocyte differentiation related-maker genes during 3T3-L1 preadipocyte differentiation. "Significant difference $(p<0.05),{ }^{*}$ Extremely significant difference $(p<0.01)$.

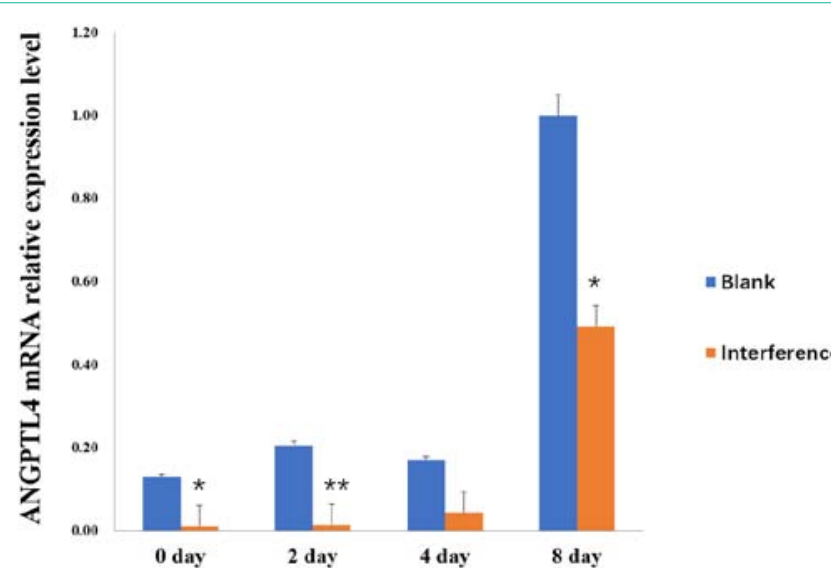

Figure 3: The silencing efficiency analysis of ANGPTL4 in 3T3-L1 preadipocyte. *Significant difference $(p<0.05)$, "Extremely significant difference $(p<0.01)$.

lipid content in white adipose tissue [35]. Therefore, ANGPTL4 is considered to be a powerful regulator of lipid metabolism and obesity [36].

The results showed that although the target gene was interfered, the differentiation of preadipocyte was not affected, indicating that lipid metabolism is a complex process which influences by multiple factors and can maintain its own balance. In the present, we found that expression of all genes except LPL (PPARG, ACAA2, SLC27A1, SCD, FASN) increased rapidly after inhibiting ANGPTL4, and then decreased significant following the increased of ANGPTL4 expression. Since all of these genes are known to be related with ANGPTL4 and affect lipid metabolism, it is preliminarily speculated that these genes play a compensatory role after the rapid decline in the disturbed expression of ANGPTL4. Instead of ANGPTL4, these genes continue to complete the differentiation process of preadipocyte. However, it was only interference in the experiment, although the interference efficiency was about $90 \%$, it was not completely knocked out, so whether the trace of the target gene continued to play a role was also a deficiency in the experiment. The specific mechanism still needs to

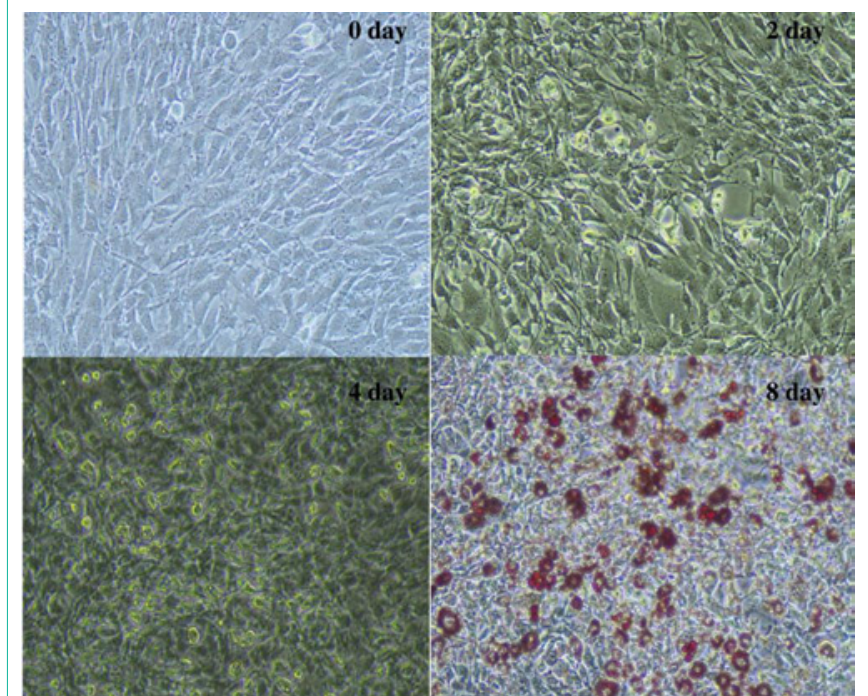

Figure 4: Differentiation of 3T3-L1 preadipocyte. (Day 8 with Oil Red O staining, 20X).

\section{Interference Group (752)}

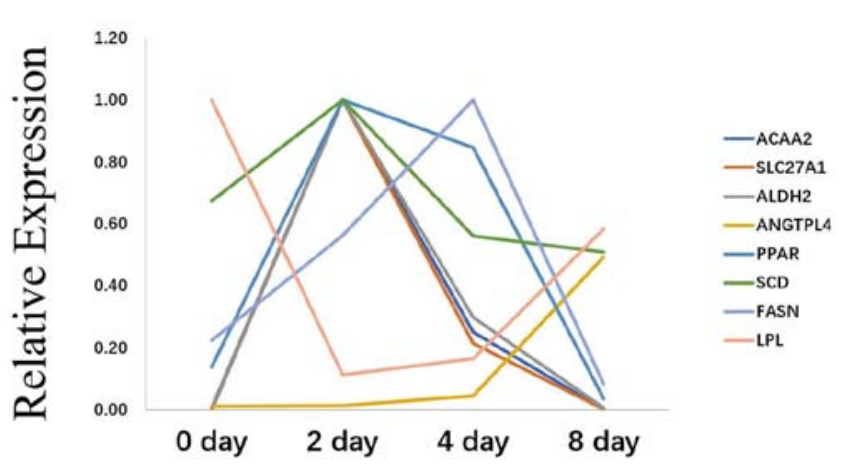

Figure 5: The effect of silencing ANGPTL4 on adipocyte differentiation related-maker genes during 3T3-L1 preadipocyte differentiation. *Significant difference $(p<0.05)$, ${ }^{*}$ Extremely significant difference $(p<0.01)$.

be explored.

After inhibiting expression of gene ANGPTL4, only the expression trend of LPL was different from that of other genes. What is worth paying attention to is whether it is related to the theory that ANGPTL4 negatively regulates LPL, which has been reported in several studies? Plasma Triglyceride (TG) levels are mainly determined by the dynamic balance between TG secreted in the small intestine and TG degradation in muscle and adipose tissue [37]. LPL is particularly important as a rate-limiting enzyme for hydrolyzing triglycerides in blood [38]. LPL is produced by muscle cells and adipocytes and is transported to the surface of endothelial cells via GPIHBP1 [39], and LPL is mainly regulated at the post-translational level [40]. ANGPTL4, as an equilibrium switch regulating homeostasis, increases blood triglyceride levels by inhibiting LPL activity [30]. ANGPTL3 and ANGPTL8, which are members of the same angiopoietin like protein family as AngPTL4, can also participate in the regulation of LPL, and their coordination 
ensures the balance of triglyceride levels. ANGPTL3 and ANGPTL4 are inhibitors of LPL activity, and AngPTL8 is inhibitors of LPL secretion [41]. Under exercise, fasting and cold exposure, ANGPTL4 inhibits LPL activity in adipose tissue, skeletal muscle and heart, inhibits LPL mediated circulating TG clearance, reduces the entry of plasma TG-derived fatty acids into adipose tissue, and improves plasma TG level, which is preferred to be absorbed by oxidized tissues [42-44]. However, this inhibition was rapidly eliminated after stable binding of GPIHBP1 with dimer form LPL [45]. Scientists found that fasting induces increased levels of AngPTL4 in adipose tissue, which reduces LPL activity by promoting protease lysis of LPL in cells [46]. ANGPTL4 derived from mouse brown adipose tissue also inhibits LPL activity and promotes thermogenesis [36]. The knockout of ANGPTL4 in mice resulted in higher body fat and body weight in mice [47]. Except him PPAR signaling pathway which ANGPTL4 is mainly involved, some scientists believe that ANGPTL4 and LPL also constitute a pathway. Scientists have found that overexpression of miR134 reduces the expression of ANGPTL4 in aortic tissues and peritoneal macrophages, while increasing the expression and activity of LPL, and promotes lipid accumulation and secretion of pro-inflammatory cytokines, thus accelerating the formation of atherosclerosis [48]. So, if they form a pathway, then when ANGPTL4 is suppressed it may block the pathway and thus inhibit the expression of LPL. Although ANGPTL4 is a negative regulator of LPL, it is also a close interaction, and when one ring is disturbed, the other will also be disturbed. When the expression of ANGPTL4 began to recover after 4 days, the expression of LPL was also restored, and the pathway constituted by them could also start to recover. Of course, this result was limited to the 3T3-L1 cell line, which still needs to be further verified.

\section{Conclusions}

In this study, we observed the changes in the expression levels of key genes before and after ANGPTL4 interference to explore the specific mechanism of ANGPTL4's influence on lipid metabolism. Results shows that, after inhibiting expression of gene ANGPTL4, only the expression trend of LPL was similar with ANGPTL4, which is different from all other genes. We suspect that, ANGPTL4 and LPL form a pathway, then when ANGPTL4 is suppressed it may block the pathway and thus inhibit the expression of LPL. Other genes play a compensating role and continue to complete the differentiation process.

\section{Author Contributions}

$\mathrm{CW}, \mathrm{KX}$, and $\mathrm{XZ}$ conceived and designed the experiments. XZ performed the experiments, analyzed the data, contributed reagents/ materials/analysis tools, and $\mathrm{XZ}$ wrote the paper. $\mathrm{CW}$ and $\mathrm{KX}$ contributed final approval of the version to be published. HA, FZ contributed to the literature search. YL, HL contributed to the data analysis.

\section{Funding}

This research was funded by the National Key R\&D Program of China (No. 2018YFD0501005), Beijing Innovation Consortium of Agriculture Research System (BAIC02-2018), and the Program of New Breed Development via Transgenic Technology (2016ZX080011006).

\section{Conflicts of Interest}

The funders and our team co-operated in the study design and decision to publish.

\section{Data Availability Statements}

The data underlying this article will be shared on reasonable request to the corresponding author.

\section{Ethics Statement}

All experimental procedures were performed according to the Guide for Animal Care and Use of Laboratory Animals of the Institutional Animal Care and Use Committee of China Agricultural University. The experimental protocol was approved by the Departmental Animal Ethics Committee of China Agricultural University (Permit No. DK996).

\section{References}

1. Galic S, Oakhill JS, Steinberg GR. 'Adipose tissue as an endocrine organ'. Molecular and Cellular Endocrinology. 2010; 316: 129-139.

2. Kershaw EE, Flier JS. 'Adipose tissue as an endocrine organ', Journal of Clinical Endocrinology and Metabolism. 2004; 89: 2548-2556.

3. Suzuki K, Inomata K, Katoh K, Kadowaki H, Shibata T. 'Genetic correlations among carcass cross-sectional fat area ratios, production traits, intramuscular fat, and serum leptin concentration in Duroc pigs'. Journal of Animal Science. 2009; 87: 2209-2215.

4. Choe SS, Huh JY. Hwang IJ, Kim JI, Kim JB. 'Adipose tissue remodeling: Its role in energy metabolism and metabolic disorders'. Frontiers in Endocrinology. 2016; 7: 30.

5. Gregoire FM, Smas CM, Sul HS. 'Understanding adipocyte differentiation'. Physiological Reviews. 1998; 78: 783-809.

6. Matsubara Y, Aoki M, Endo T, Sato K. 'Characterization of the expression profiles of adipogenesis-related factors, ZNF423, KLFs and FGF10, during preadipocyte differentiation and abdominal adipose tissue development in chickens'. Comparative Biochemistry and Physiology - B Biochemistry and Molecular Biology. 2013; 165: 189-195.

7. Cawthorn WP, Sethi JK. 'TNF- $\alpha$ and adipocyte biology'. FEBS Letters. 2008; 582: 117-131.

8. Lane MD, Tang QQ, Jiang MS. 'Role of the CCAAT enhancer binding proteins (C/EBPs) in adipocyte differentiation'. Biochemical and Biophysical Research Communications. 1999; 266: 677-683.

9. Ricote M, Li AC, Willson TM, Kelly CJ, Glass CK. 'The peroxisome proliferatoractivated receptor-y is a negative regulator of macrophage activation'. Nature. 1998; 391: 79-82

10. Picard F, Kurtev M, Chuang N, Topark-Ngarm A, Senawong T, De Oliveira $\mathrm{RM}$, et al. 'Sirt1 promotes fat mobilization in white adipocytes by repressing PPAR-y'. Nature. 2004; 429: 771-776.

11. Lee D, Goldberg AL. 'SIRT1 protein, by blocking the activities of transcription factors FoxO1 and FoxO3, inhibits muscle atrophy and promotes muscle growth'. Journal of Biological Chemistry. 2013; 288: 30515-30526.

12. Green $\mathrm{H}$, Meuth M. 'An established pre-adipose cell line and its differentiation in culture'. Cell. 1974; 3: 127-133.

13. Green $\mathrm{H}$, Kehinde $\mathrm{O}$. 'An established preadipose cell line and its differentiation in culture II. Factors affecting the adipose conversion'. Cell. 1975; 5: 19-27.

14. Ahn J, Lee H, Kim S, Park J, Ha T. 'The anti-obesity effect of quercetin is mediated by the AMPK and MAPK signaling pathways'. Biochemical and Biophysical Research Communications. 2008; 373: 545-549.

15. Sun T, Fu M, Bookout AL, Kliewer SA, Mangelsdorf DJ. 'MicroRNA let-7 regulates 3T3-L1 adipogenesis'. Molecular Endocrinology. 2009; 23: 925931. 
16. Tan Y, Gan M, Fan Y, Li L, Zhong Z, Li X, et al 'miR-10b-5p regulates 3T3-L1 cells differentiation by targeting Apol6'. Gene. 2019; 687: 39-46.

17. Liu H, Li H, Jin L, Li G, Hu S, Ning C, et al. 'Long Noncoding RNA GAS5 Suppresses 3T3-L1 Cells Adipogenesis Through miR-21a-5p/PTEN Signal Pathway'. DNA and Cell Biology. 2018; 37: 767-777.

18. Pruszyńska-Oszmałek E. 'Kisspeptin-10 inhibits proliferation and regulates lipolysis and lipogenesis processes in 3T3-L1 cells and isolated rat adipocytes'. Endocrine. 2017; 56: 54-64.

19. Yoon JC. 'Peroxisome Proliferator-Activated Receptor gamma Target Gene Encoding a Novel Angiopoietin-Related Protein Associated with Adipose Differentiation'. Molecular and Cellular Biology. 2000; 20: 5343-5349.

20. Mikhak B. 'Angiopoietin-like 4 (ANGPTL4) gene polymorphisms and risk of brain arteriovenous malformations'. Cerebrovascular Diseases. 2011; 31 338-345.

21. Gomez Perdiguero E. 'ANGPTL4- $\alpha$ $\beta 3$ interaction counteracts hypoxiainduced vascular permeability by modulating Src signaling downstream of vascular endothelial growth factor receptor 2'. The Journal of Pathology. 2016; 240: 461-471.

22. Xu A. 'Angiopoietin-like protein 4 decreases blood glucose and improves glucose tolerance but induces hyperlipidemia and hepatic steatosis in mice'. Proceedings of the National Academy of Sciences of the United States of America. 2005; 102: 6086-6091.

23. Bini S. 'The Interplay between Angiopoietin-Like Proteins and Adipose Tissue: Another Piece of the Relationship between Adiposopathy and Cardiometabolic Diseases?' International Journal of Molecular Sciences. 2021; 22: 742

24. Kim HK. 'Hypothalamic Angpt/4/Fiaf is a novel regulator of food intake and body weight'. Diabetes. 2010; 59: 2772-2780.

25. Goh YY. 'Angiopoietin-like 4 interacts with integrins $\beta 1$ and $\beta 5$ to modulate keratinocyte migration'. American Journal of Pathology. 2010; 177: 2791 2803.

26. Lichtenstein L. 'Angpt|4 protects against severe proinflammatory effects of saturated fat by inhibiting fatty acid uptake into mesenteric lymph node macrophages'. Cell Metabolism. 2010; 12: 580-592.

27. Kersten S. 'Characterization of the fasting-induced adipose factor FIAF, novel peroxisome proliferator-activated receptor target gene'. Journal of Biological Chemistry. 2000; 275: 28488-28493.

28. Feng SQ, et al. 'Cloning, chromosome mapping and expression characteristics of porcine ANGPTL3 and -4'. Cytogenetic and Genome Research. 2006; 114 44-49.

29. Yang L, et al. 'Angiopoietin-Like Protein 4 Is a High-Density Lipoprotein (HDL) Component for HDL Metabolism and Function in Nondiabetic Participants and Type-2 Diabetic Patients'. Journal of the American Heart Association. 2017; 6: e005973.

30. Yoshida K, et al. 'Angiopoietin-like protein 4 is a potent hyperlipidemiainducing factor in mice and inhibitor of lipoprotein lipase'. Journal of Lipid Research. 2002; 43: 1770-1772.

31. Qiao L, et al. 'Obesity Reduces Maternal Blood Triglyceride Concentrations by Reducing Angiopoietin-Like Protein 4 Expression in Mice'. Diabetes. 2020 69: 1100-1109.
32. Zhao $X$, et al. 'Transcriptome analysis of landrace pig subcutaneous preadipocytes during adipogenic differentiation'. Genes. 2019; 10: 552

33. Kahn B, Flier J. 'Obesity and insulin resistance'. The Journal of Clinical Investigation. 2000; 106: 473-481.

34. Mourot J, Hermier D. 'Lipids in monogastric animal meat'. Reproduction Nutrition Development. 2001; 41: 109-118.

35. Chen YQ. 'Angiopoietin-like protein 4 (E4OK) and ANGPTL4/8 complex have reduced, temperature-dependent LPL-inhibitory activity compared to ANGPTL4'. Biochemical and Biophysical Research Communications. 2021; 534: 498-503.

36. Singh AK. 'Brown adipose tissue derived ANGPTL4 controls glucose and lipid metabolism and regulates thermogenesis'. Molecular Metabolism. 2018; 11 : 59-69.

37. Rosen ED, MacDougald OA. 'Adipocyte differentiation from the inside out'. Nature Reviews Molecular Cell Biology. 2006; 7: 885-896.

38. Yagyu $H$. 'Lipoprotein lipase $(L p L)$ on the surface of cardiomyocytes increases lipid uptake and produces a cardiomyopathy'. Journal of Clinical Investigation. 2003; 111: 419-426.

39. Davies BSJ, et al. 'GPIHBP1 is responsible for the entry of lipoprotein lipase into capillaries'. Cell Metabolism. 2010; 12: 42-52.

40. Ogata HY, Oku H. 'The effects of dietary retinoic acid on body lipid deposition in juvenile red sea bream (Pagrus major); a preliminary study'. Aquaculture. 2001; 193: 271-279.

41. Kovrov O. 'On the mechanism of angiopoietin-like protein 8 for control of lipoprotein lipase activity'. Journal of Lipid Research. 2019; 60: 783-793.

42. Kroupa O. 'Linking nutritional regulation of Angpt|4, Gpihbp1, and Lmf1 to lipoprotein lipase activity in rodent adipose tissue'. BMC Physiology. 2012; 12: 13.

43. Catoire M. 'Fatty acid-inducible ANGPTL4 governs lipid metabolic response to exercise'. Proceedings of the National Academy of Sciences. 2014; 111 : E1043-E1052.

44. Dijk W, Heine M, Vergnes L, Boon MR, Schaart G, Hesselink MKC, et al. 'ANGPTL4 mediates shuttling of lipid fuel to brown adipose tissue during sustained cold exposure'. Elife. 2015; 4: 1-13.

45. Sonnenburg WK, Yu D, Lee EC, Xiong W, Gololobov G, Key B, et al. 'GPIHBP1 stabilizes lipoprotein lipase and prevents its inhibition by angiopoietin-like 3 and angiopoietin-like 4'. Journal of Lipid Research. 2009; 50: 2421-2429.

46. Dijk W, Ruppert PMM, Oost LJ, Kersten S. 'Angiopoietin-like 4 promotes the intracellular cleavage of lipoprotein lipase by PCSK3/furin in adipocytes'. Journal of Biological Chemistry. 2018; 293: 14134-14145

47. Mattijssen F, Alex S, Swarts $\mathrm{nHJ}$, Groen AK, van Schothorst EM, Kersten S. 'Angpt/4 serves as an endogenous inhibitor of intestinal lipid digestion'. Molecular Metabolism. 2014; 3: 135-144.

48. Ye Q, Tian GP, Cheng HP, Zhang X, Ou X, YuXH, et al. 'MicroRNA-134 promotes the development of atherosclerosis via the angpt/4/Ipl pathway in apolipoprotein e knockout mice'. Journal of Atherosclerosis and Thrombosis. 2018; 25: 244-253 